Revista de
Economild
Contemporâned

\title{
EFEITOS DA POLÍTICA DE SALÁRIO MÍNIMO SOBRE O MERCADO DE TRABALHO METROPOLITANO: UMA ANÁLISE EMPÍRICA A PARTIR DE VETORES AUTORREGRESSIVOS (VAR) - (2003-2015)
}

\author{
Girleno Costa Pereira ${ }^{a}$ \\ André de Souza Melo ${ }^{b}$ \\ Leonardo Ferraz Xavier

\begin{abstract}
${ }^{a}$ Mestre pelo Programa de Pós-Graduação em Administração e Desenvolvimento Rural (PADR) da Universidade Federal Rural de Pernambuco (UFRPE). Administração e Desenvolvimento Rural (PADR/UFRPE).

'Professor do Departamento de Economia da UFRPE.
\end{abstract} \\ bProfessor do Departamento de Economia da UFRPE e Coordenador do Programa de Pós-Graduação em
}

Artigo recebido em 19/01/2016 e aceito para publicação em 05/03/2017.

RESUMO: Com intuito de verificar os efeitos que a política de salário mínimo exerce sobre o mercado de trabalho metropolitano brasileiro, o presente estudo contribui ao verificar a sua inter-relação com variáveis como taxa de atividade, grau de informalidade e taxa de desemprego em quatro regiões metropolitanas (Recife, Porto Alegre, Belo Horizonte e São Paulo), separadamente, no período de janeiro de 2003 a março de 2015. Ao utilizar o método de autorregressão vetorial (VAR), os resultados mostram que a relação entre salário mínimo e taxa de atividade se deu com ingresso de mão de obra apenas em uma região estudada. Nas demais regiões observam-se tanto uma tendência de queda no grau de informalidade quanto um aumento na taxa de desemprego.

PALAVRAS-CHAVE: salário mínimo; mercado de trabalho; VAR.

CLASSIFICAÇÃO JEL: C01; E24. 


\title{
EFFECTS OF THE MINIMUM WAGE POLICY ON THE METROPOLITAN LABOR MARKET: AN EMPIRICAL ANALYSIS FROM VECTORS AUTOREGRESSIVE (VAR) - (2003-2015)
}

\begin{abstract}
In order to verify the effects of the minimum wage policy has on the Brazilian metropolitan labor market, this study adds to check their interrelation with variables such as activity rate, degree of informality and unemployment rate in four Regions metropolitan (Recife, Porto Alegre, Belo Horizonte and São Paulo), separately, from January 2003 to March 2015. By using the vector autoregression method (VAR), the results show that the relationship between the minimum wage and activity rate occurred with labor ticket in just a study area. In the other regions are observed both a downward trend in the degree of informality as an increase in the unemployment rate.
\end{abstract}

KEYWORDS: minimum wage; labor market; VAR. 


\section{INTRODUÇÃO}

Variações no nível do salário mínimo podem ter uma multiplicidade de efeitos sobre o funcionamento da economia em geral. Esses efeitos tendem a ser multifacetários, conjugando impactos positivos em algumas dimensões e negativos em outras (FOGUEL, ULYSSEA e COURSEIL, 2014). Vários estudos têm procurado avaliar os custos e benefícios das políticas de salário mínimo que vêm sendo implementadas em vários países. Na maior parte das ocasiões, são os impactos do salário mínimo sobre variáveis tais como nível de emprego e salário que têm provocado os mais intensos debates (SOUZA e TARGINO, 2005).

No Brasil, a discussão sistemática acerca dos efeitos do salário mínimo sobre o mercado de trabalho remonta pelo menos à década de 1970, sendo desencadeada pela redução continuada do salário real em decorrência de medidas de arrocho salarial adotadas pelo governo no período entre os anos de 1960 e 1970 e, ao mesmo tempo, pelo agravamento dos indicadores de desigualdade de renda, direcionando a discussão sobre o tema para a possível relação entre os dois acontecimentos (GANDRA, 2004).

Conforme observa Lemos (2004), uma política de manutenção do valor real do salário mínimo faz com que o valor nominal deste e do rendimento mensal dos trabalhadores sofram elevações que garantam o poder de compra dos trabalhadores. Porém, o nível geral de salários, assim como o valor do salário mínimo, é uma variável de grande importância para a economia, pois mudanças em seu valor tendem a se propagar e seus impactos afetam, em maiores ou menores proporções, as variáveis econômicas.

No que tange aos efeitos diretos, o salário mínimo tende a gerar modificações sobre o nível e dispersão salarial, assim como sobre o nível e estrutura do emprego do setor formal do mercado de trabalho. Quando esse mercado como um todo é influenciado pelo processo de ajuste no setor formal, aparecem os efeitos indiretos do salário mínimo, atingindo, essencialmente, todos os indicadores do emprego ao impacto da variação no valor do mínimo. Assim, a conjunção dos efeitos diretos e indiretos do salário mínimo pode causar impacto no equilíbrio do mercado de trabalho por inteiro, com possíveis reflexos sobre um conjunto de variáveis, como a taxa de desemprego, a taxa de atividade, o grau de formalização, composição do emprego, entre outros (FOGUEL, ULYSSEA e COURSEIL, 2014).

A fim de contribuir para as investigações acerca do papel do salário mínimo sobre o mercado de trabalho brasileiro, o objetivo do estudo é a análise dos impactos do salário real sobre variáveis de emprego nas regiões metropolitanas de Recife, Porto Alegre, Belo Horizonte e São Paulo no período de janeiro de 2003 a março de 2015. A escolha de variáveis como a taxa de atividade, o grau de informalidade e a taxa de desemprego ocorreu de modo a representar o estado de ocupação e desocupação da mão de obra nessas regiões. 


\section{FUNDAMENTAÇÃO TEÓRICA: SALÁRIO MÍNIMO E MERCADO DE TRABALHO}

De acordo com Staduto (2002), a teoria clássica trouxe grandes contribuições e lançou as bases para as teorias mais modernas sobre mercados de trabalho e salários. Os fundamentos clássicos deste mercado foram, até recentemente, aplicados em alguns modelos de desenvolvimento econômico. No entanto, esta teoria não tem sido aplicada de forma mais contundente para estimar os modelos acerca de salários. A teoria neoclássica, em competição perfeita no mercado de trabalho, pode ser vista como uma evolução dos modelos clássicos e, portanto, ela é mais utilizada na análise dos salários, principalmente nos setores mais concorrenciais da economia.

A sua origem teve como ponto de partida a publicação de um artigo de Hicks (1937) sobre as pressuposições teóricas de John Maynard Keynes no seu livro Teoria Geral do Emprego, do Juro e da Moeda, publicado em 1936. A partir das críticas de Hicks a Keynes, a abordagem analítica sobre o mercado de trabalho tornou-se alvo das atenções nos debates teóricos. Na Teoria Geral, Keynes fazia um ataque aos postulados dos autores que ele denominava como clássicos. Entretanto, apesar da tentativa de Keynes de diferenciar a sua teoria em relação à dos autores clássicos, para Hicks (1937), ela era essencialmente a mesma, sendo apenas um caso particular, quando ocorresse rigidez salarial acima do salário de equilíbrio.

Dessa forma, a contribuição de Keynes seria mais limitada, restringindo-se à construção de uma teoria que relaciona o "lado real" da economia com o "lado monetário" (STADUTO, 2002). Nesse cenário, em Foguel, Ulyssea e Courseil (2014), tem-se que variações no nível do salário mínimo podem afetar o funcionamento da dinâmica do mercado de trabalho. Tal afirmação deve-se ao fato de que o salário mínimo é um preço importante nesse mercado, o que implica que mudanças no seu valor tendem a se propagar.

Lemos (2004) destaca que uma das variáveis mais afetadas é o nível de emprego, ou seja, aumentos no salário mínimo podem comprimir a distribuição dos salários na economia, causando, portanto, alterações nesse nível. Evidentemente, a magnitude dos impactos do salário mínimo vai depender dos tipos de efeitos provocados, independentemente da variável de trabalho, além de uma série de outros condicionantes.

Muito embora as teorias reverberem sobre o papel que o salário mínimo exerce no mercado de trabalho, autores como Macedo e Garcia (1978) julgavam que na década de 1970 a relevância do salário mínimo vinha decrescendo em alguns segmentos do mercado de trabalho, não provocando mais tantos efeitos na remuneração da força de trabalho não qualificada. Para esses autores, em uma situação de rápido crescimento econômico, a taxa de salário se descola do salário mínimo caso este seja reajustado em um ritmo inferior à inflação. 
Em réplica ao trabalho de Macedo e Garcia (1978), autores como Giambiagi e Franco (2007) argumentam que existem três efeitos que as políticas de salário mínimo desempenham:

O primeiro é sua natureza compulsória, uma vez que, teoricamente, no mercado formal a legislação obriga o empregador a obedecer a parâmetros fixados institucionalmente, seja pela Constituição ou pela lei. O segundo é o caráter de indexador da variável, que funciona como piso para outras remunerações que não as recebidas no mercado de trabalho - casos do seguro-desemprego e do piso previdenciário e assistencial. O terceiro canal, finalmente, é o assim denominado "efeito farol", que consiste no poder de balizamento da figura do salário mínimo incidindo sobre o mercado informa. (GIAMBIAGI e FRANCO, 2007, p. 11)

Barros e Carvalho (2005) acrescentam aos efeitos da política de salário mínimo o que denominaram efeito "enclausuramento", que está associado ao crescimento do setor não coberto da economia, ou seja, o setor informal. Para melhor compreensão, pode-se considerar o caso de uma mão de obra heterogênea inserida em um mercado de trabalho segmentado, no qual ocorre uma elevação do salário mínimo. Nesta situação, haveria um aumento dos salários dos trabalhadores pouco qualificados no setor formal sem o respectivo aumento de produtividade, conduzindo a uma redução nos salários de todos os trabalhadores, e não apenas dos menos qualificados.

Romannatto, Porcile e Curado (2008) sugerem que dentro da relação entre emprego e salários podemos encontrar uma situação em específico em que uma queda nesse mercado pode não significar queda nos ganhos salariais dos trabalhadores. Dentro da economia brasileira, tal fenômeno pode ser explicado por conta da liberalização comercial, do aumento da concorrência e da introdução de novas formas de organização do trabalho, juntamente com o comportamento da taxa de câmbio na década de 1990.

Cacciamalli (2005) argumenta que o os efeitos do aumento do salário mínimo vêm no sentido de diminuição na demanda por mão de obra no mercado de trabalho formal, ocasionando transitoriedade dos trabalhadores desempregados em direção à informalidade. A procura por emprego na informalidade reduz o salário real deste setor, gerando deslocamento na curva de oferta de trabalho no mercado informal. A transição entre mercado formal para o informal é causada pela elevação do salário mínimo, que beneficia os trabalhadores formais que continuam empregados em detrimento dos trabalhadores desocupados em busca por empregos com menores salários reais no setor informal da economia.

A diminuição na demanda no mercado formal de trabalho tratada por Cacciamalli (2005) já havia sido abordada em outro momento nos trabalhos de Welch (1974), 
segundo o qual a elevação do salário mínimo faz aumentar o salário do setor formal. Esse setor se ajustará à elevação do mínimo da mesma maneira que o modelo padrão, isto é, as firmas demitirão uma parte de seus trabalhadores devido à mudança no preço relativo dos fatores. Os trabalhadores têm a opção de irem para o setor informal ou então saírem da força de trabalho. Os que tiveram salário de reserva acima do novo salário do setor informal podem decidir não trabalhar neste setor, ou seja, alguns trabalhadores que perderam o emprego no setor formal (assim como alguns que estavam empregados no setor informal) podem decidir sair da força de trabalho, já que o salário do setor informal pode ficar situado abaixo dos seus salários de reserva.

Contudo, ao considerar somente os impactos do salário mínimo sobre os setores formal e informal, Welch (1974) omite os possíveis efeitos que o salário mínimo pode ter sobre o desemprego. Autores do mesmo período como Mincer (1976) e Gramlich (1976) ampliaram uma série de outras possibilidades em termos da direção que os fluxos da mão de obra podem ter a partir de uma variação no valor do salário mínimo. Deste modo, admite-se que os trabalhadores demitidos do setor formal pela imposição do mínimo fluam para o estado de desemprego para esperar por uma oportunidade de emprego naquele setor que oferece a melhor remuneração.

O impacto do salário mínimo sobre o desemprego depende de uma série de fatores. Do ponto de vista tecnológico, quanto mais a mão de obra é remunerada próximo ao valor do salário mínimo, maior será o impacto de reajustes salariais sobre o desemprego. É o que se observa em trabalhadores menos qualificados, quem possuem alto grau de substituição. O resultado final também pode ser influenciado por fatores institucionais. Por exemplo, a atuação dos sindicatos pode obrigar as empresas a não demitirem os trabalhadores que seriam beneficiados pela imposição do salário mínimo. Por fim, o impacto dependerá também da situação da economia, uma vez que um reajuste do salário mínimo deve impactar mais fortemente o mercado de trabalho quanto mais próximo o salário mínimo estiver do rendimento médio dos empregados (BOERI, GARIBALDI e RIBEIRO, 2010).

Em países desenvolvidos, conforme Freitas (2011), o salário mínimo não exerce influência significativa no mercado de trabalho, desde que seja fixado em um nível abaixo do salário de equilíbrio. Já em países em desenvolvimento, como o Brasil, a teoria prevê que reajustes do salário mínimo acima do crescimento da produtividade do trabalhador têm consequências sobre o nível de emprego, uma vez que é comum parcela não desprezível da população ganhando em torno do piso salarial. Ainda assim, o autor acrescenta que os efeitos do salário mínimo sobre este mercado, independentemente do grau de desenvolvimento do país, terão efeitos diferenciados a partir da situação do trabalhador.

Para aqueles empregados que já ganhavam acima do novo piso estabelecido, não há efeito direto do salário mínimo. Vale ressaltar, entretanto, que pode haver impactos indi- 
retos, com demissão de trabalhadores não qualificados e contratação de trabalhadores mais qualificados. Esse impacto indireto, contudo, é difícil de ser estabelecido em um primeiro momento, pois depende do grau de substituição entre capital, trabalho qualificado e trabalho não qualificado. Já os trabalhadores cujo salário era menor do que o novo mínimo e que conseguiram manter seus empregos no setor formal obtêm um ganho de bem-estar, pois passam a ter maior remuneração. Por fim, os trabalhadores cujo salário era inferior ao novo mínimo e que perderam seus empregos no setor formal tornam-se desempregados ou migram para o setor informal (FREITAS, 2011).

Moura e Neri (2008), ao observarem o papel que o salário mínimo exerce na heterogênea economia brasileira, afirmam que a aplicação de um piso salarial representa oportunidade privilegiada de avaliação dos impactos dessa variável salarial no momento em que baliza, simultaneamente, ativos e inativos dos setores público e privado do País. Os autores tratam esses setores como passíveis de diferentes efeitos a partir da fixação salarial, com distintas magnitudes.

Para Soares (2004), a estipulação de um piso perante o salário mínimo poderia gerar ainda dois efeitos opostos com um maior incentivo ao descumprimento, pois o ganho do subpagamento é maior do que o custo esperado e há uma maior probabilidade de os trabalhadores denunciarem os empregadores, o que aumentaria os índices de desemprego, sendo o segundo mais efetivo devido à baixa efetividade da lei.

Em suma, as variações no salário mínimo têm diferentes impactos sobre uma diversidade de indicadores de desempenho do mercado de trabalho independentemente do setor, seja ele público ou privado. Mesmo considerando seus efeitos, não é possível dizer a priori qual é a direção final dessas alterações. Analogamente, dependendo da magnitude do impacto do salário mínimo sobre os diversos setores de atividade econômica, é possível que a taxa de emprego de um determinado setor aumente enquanto a de outro diminui (FOGUEL, ULYSSEA e COURSEIL, 2014).

\section{PROCEDIMENTOS METODOLÓGICOS}

Para estimar os impactos do salário mínimo sobre o mercado de trabalho metropolitano brasileiro, utiliza-se um modelo de autorregressão vetorial (VAR), a partir da base de dados da PME - Pesquisa Mensal do Emprego (IBGE, 2015) e IPEA (2015) para quatro regiões metropolitanas do Brasil (Recife, Porto Alegre, Belo Horizonte e São Paulo) separadamente, no período de janeiro de 2003 a março de 2015. Tais regiões metropolitanas foram escolhidas para representar as regiões incluídas na PME (Nordeste, Sudeste e Sul), de modo que, acredita-se, apresentam efeitos positivos, nulos ou negativos no nível de emprego. 


\subsection{DADOS UTILIZADOS}

A base de dados a ser utilizada consiste da coleta de séries mensais obtidas junto a instituições como o IPEA (Instituto de Pesquisa Econômica Aplicada) para o salário mínimo real e o grau de informalidade ${ }^{1}$ e ao IBGE (Instituto Brasileiro de Geografia e Estatística) para séries do mercado de trabalho de cada região metropolitana separadamente como a taxa de atividade e a taxa de desemprego.

\subsection{MODELO EMPÍRICO: A ESTRUTURA DO VETOR AUTORREGRESSIVO}

Por meio do uso da metodologia VAR, busca-se analisar as inter-relações do salário mínimo nos distintos modelos propostos. Segundo Sims (1980), os modelos multiequacionais se tornam capazes de analisar as inter-relações entre as variáveis macroeconômicas e seus efeitos a partir de "choques" que provocam ciclos na economia, isto é, esses modelos foram capazes de analisar a importância relativa de cada "surpresa" (ou inovações) sobre as variáveis do sistema macroeconômico. É a abordagem empírica que possibilita maior entendimento de como as variáveis macroeconômicas respondem a esses "choques" simultaneamente.

Dessa forma, admite-se que o ponto de partida para estudos de modelos multivariados é tratar todas as variáveis simetricamente. Será então utilizado um sistema de equações nas quais se assume que as variáveis sejam interdependentes e estejam relacionadas por uma memória autorregressiva (Enders, 1995). No presente trabalho, o vetor $X_{t}$ compreende as variáveis: salário mínimo (SM), taxa de atividade $(A T V)$, grau de informalidade $(I N F)$, taxa de desemprego $(D E S)$. O sistema de equações é:

$$
\begin{aligned}
& S M_{t j}=b_{10}+b_{11} S M_{(t-k) j}+b_{12} A T V_{(t-i) j}+b_{13} I N F_{(t-i) j}+b_{14} D E S_{(t-i) j}+\varepsilon_{1 t} \\
& A T V_{t j}=b_{20}+b_{21} S M_{(t-i) j}+b_{22} A T V_{(t-k) j}+b_{23} I N F_{(t-i) j}+b_{24} D E S_{(t-i) j}+\varepsilon_{2 t} \\
& I N F_{t j}=b_{30}+b_{31} S M_{(t-i) j}+b_{32} A T V_{(t-i) j}+b_{33} I N F_{(t-k) j}+b_{34} D E S_{(t-i) j}+\varepsilon_{3 t} \\
& D E S_{t j}=b_{40}+b_{41} S M_{(t-i) j}+b_{42} A T V_{(t-i) j}+b_{43} I N F_{(t-i) j}+b_{44} D E S_{(t-k) j}+\varepsilon_{4 t} \\
& k=1,2,3, \ldots, p \\
& i=0,1,23, \ldots, p \\
& j=j \text {-ésima região metropolitana }
\end{aligned}
$$

\footnotetext{
O grau de informalidade aqui utilizado é definido como o grau I, obtido com base nos microdados da Pesquisa Nacional por Amostra de Domicílios do IBGE. Essa taxa corresponde ao resultado da seguinte divisão: (empregados sem carteira + trabalhadores por conta própria)/(trabalhadores protegidos + empregados sem carteira + trabalhadores por conta própria) (IPEA, 2015).
} 
Tal sistema não pode ser estimado por mínimos quadrados ordinários, pois permite que os choques possam ter efeitos contemporâneos diretos e indiretos sobre as variáveis (viés de equação simultânea). Para contornar esse problema, transformou-se esse sistema de equações primitivo em uma forma chamada de forma padrão. Enders (1995) sugere transformar o sistema de equações em um vetor autorregressivo da seguinte forma:

$$
B X_{t}=\Gamma_{0} \sum_{i=1}^{p} \Gamma_{i} x_{t-i}+\varepsilon_{t}
$$

Em (2), $B$ representa a matriz dos parâmetros contemporâneos, $\Gamma_{0}$ é um vetor de interceptos, $\Gamma_{i}$ é a matriz dos parâmetros das variáveis no tempo $t-i(i=1,2, \ldots, p)$. Pré-multiplicando a equação anterior por $B$-1, obtém-se o modelo VAR na forma padrão:

$$
X_{t}=A_{o} \sum_{i=0}^{p} A_{i} x_{t-i}+\varepsilon_{t}
$$

Em (3), $A_{0}=B^{-1} \Gamma_{0}, A_{i}=B^{-1} \Gamma_{i}$ e $\varepsilon_{t}=B^{-1} \varepsilon_{t} X_{t}$ é um vetor $(n \times 1)$ contendo as variáveis a serem incluídas no VAR; $A_{0}$ é um vetor $(\boldsymbol{n} \times 1)$ de interceptos; $A_{i}$ são matrizes $(n \times n)$; e $\varepsilon_{t}$ é um vetor $(\mathrm{n} \times 1)$ de termos de erros com média zero, variância constante e não correlacionados. Dadas essas características, o modelo VAR pode ser estimado por mínimos quadrados ordinários. Para construir a função de impulso-resposta do VAR, é necessário que se represente a série autorregressiva em um modelo em termos de médias móveis (VMA). Essa conversão de um VAR para um VMA é possível desde que o sistema seja estável, respeitando a propriedade de invertibilidade do modelo de Box-Jenkins, ou seja, todas as raízes da matriz dos coeficientes tenham módulo menor que 1 . Dito de outra forma, o modelo $y_{t}=a_{0}+a_{1} y_{t-1}+\varepsilon_{t}$ exige que $a_{1}$ seja menor que 1 em módulo, apresentando, assim, a condição de estacionariedade.

Um modelo vetorial representado por médias móveis é descrito como:

$$
x_{t}=\mu+\sum_{t=0}^{\infty} A^{i} \varepsilon_{t-i} \text { onde } \mu=\left(I_{-} k-A_{-} 1\right)^{-1} v
$$

Sob a hipótese de que os termos de erro possuem $\mathrm{E}\left(\varepsilon_{t}\right)=0$, $\operatorname{Var}\left(\varepsilon_{t}\right)=\sigma^{2}$ e Cov $\left(\varepsilon_{t}\right)=0$, a interação entre as variáveis passa a ser expressa por:

$$
x_{t}=\mu+\sum_{i=0}^{\infty} \Phi_{i} \varepsilon_{t-i}
$$

Os coeficientes de $\Phi_{i}$ são os coeficientes da função impulso-resposta, observados a partir de choques $\varepsilon_{t-i}$ nas variáveis do modelo considerado. 


\section{SALÁRIO MÍNIMO E MERCADO DE TRABALHO NAS REGIÕES METROPOLITANAS: RESULTADOS E DISCUSSÃO A PARTIR DA ESTIMAÇÃO DO MODELO EMPÍRICO}

Esta seção apresenta os resultados do impacto do salário mínimo sobre o nível e a estrutura do emprego metropolitano. Apresenta-se, primeiramente, a relação das variáveis utilizadas e as respectivas nomenclaturas adotadas:

$\mathrm{SM}=$ valor do salário mínimo real

$\mathrm{ATV}=$ taxa de atividade

$\mathrm{INF}=$ grau de informalidade

DES = taxa de desemprego

\subsection{TESTES DE RAIZ UNITÁRIA}

Para a utilização da metodologia VAR, deve-se observar a estacionariedade das séries. Para tanto, empregou-se o teste ADF de raiz unitária.

Tabela 1 - Teste de estacionariedade de Dickey-Fuller Aumentado (ADF)

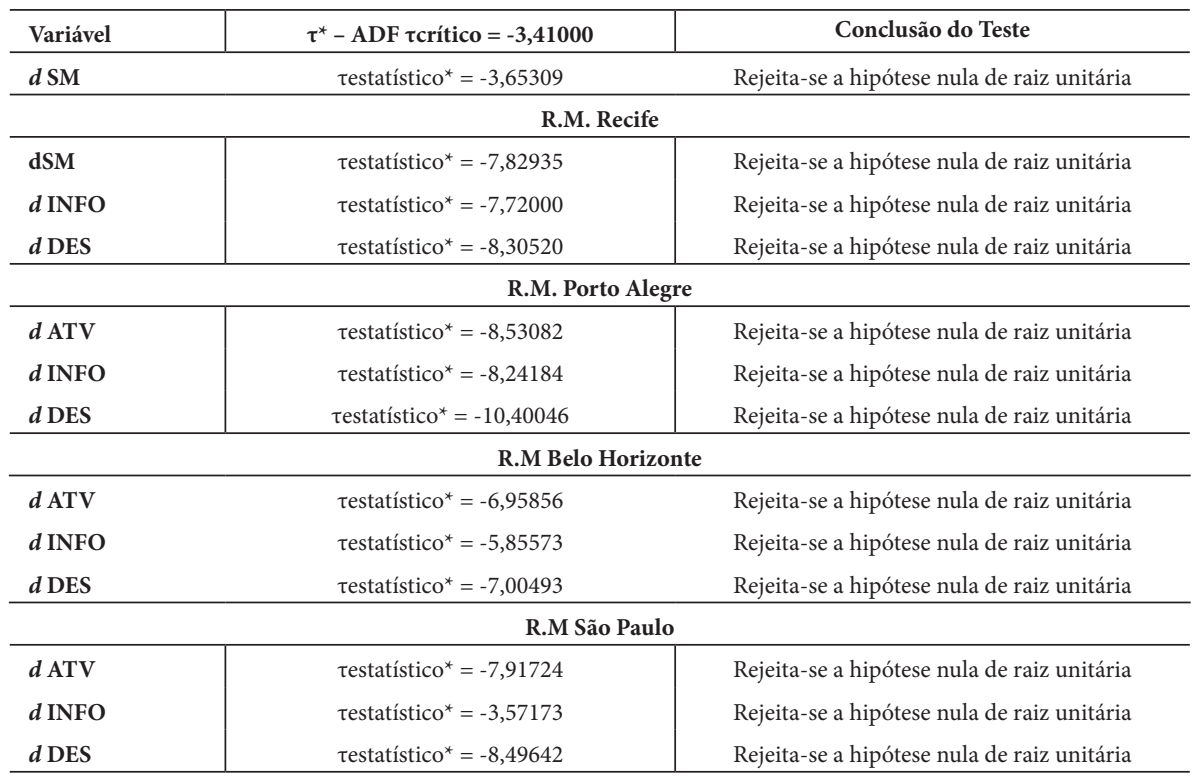

Nota: * Significativo ao nível de 5\% de significância (valores críticos em Fuller [1976] e Dickey- Fuller

[1981]); Modelo: $\Delta \Delta x_{t}=y \Delta x_{t-1}+\sum_{i=1}^{k} \lambda_{t} \Delta \Delta x_{t-1}+\varepsilon_{t}$ definido depois de constatada a não existência de termos deterministas.

Fonte: Elaboração própria com base nos dados da pesquisa. 
O teste de Dickey-Fuller (1981) Aumentado evidencia que as variáveis são não estacionárias em nível, sendo necessário diferenciá-las para que os erros se tornem ruído branco. Para as séries em nível, os resultados dos testes de Dickey-Fuller para raiz unitária indicaram que as variáveis SM (salário mínimo real), ATV (taxa de atividade), INF (grau de informalidade) e DES (taxa de desemprego) são não estacionárias. Contudo, aplicando-se o teste para estas em primeiras diferenças constatou-se a rejeição da hipótese nula de raiz unitária ao nível de 5\%. Logo, as séries econômicas SM, ATV, INFO e DES são integradas de ordem 1, I (1). Os resultados dos testes de Dickey-Fuller para raiz unitária, com constante e tendência, são apresentados na Tabela 1.

\subsection{TESTES DE COINTEGRAÇÃO}

Para Engle-Granger (1981), se duas séries não estacionárias formarem um vetor de coeficientes que gerem resíduos estacionários, diz-se que estas séries cointegram. Os resultados dos testes de raízes unitárias aplicados, individualmente, às séries estudadas, rejeitam as hipóteses nulas de que as séries são integradas de ordem, I (0), o que significa dizer que as séries são integradas de ordem um, I (1), porque cada uma delas possui raiz unitária. A resposta apresentada na Tabela I (anexo) indica que a hipótese nula da raiz unitária para os resíduos é rejeitada, o que sugere que existe uma relação de cointegração entre as quatro séries temporais de salário mínimo real, taxa de atividade, grau de informalidade e taxa de desemprego, implicando a existência de um equilíbrio de longo prazo entre elas.

\subsection{TESTE F PARA CAUSALIDADE DE GRANGER}

Nos testes de causalidade de Granger (GRANGER, 1969), o VAR é identificado por meio de uma decomposição de Cholesky na qual a ordenação causal é definida em conformidade com os resultados de testes de causalidade de Granger, sob a hipótese implícita de que a ausência de causalidade (no sentido de Granger) de uma variável $X$ para outra variável $Z$ deve implicar a ausência de efeito contemporâneo de $X$ sobre $Z$.

Efetuado o teste conforme a Tabela II (anexo), não se encontrou relação significativa de causalidade no sentido de Granger entre as variáveis, em nível de significância de 5\%. Contudo, isso não constitui um problema, visto que o objetivo aparente desse procedimento seria reduzir o grau de arbitrariedade na escolha da ordenação causal a ser adotada, pois esta estaria amparada em critérios estatísticos. Mas trata-se de um equívoco, pois o conceito de causalidade de Granger não guarda necessariamente nenhuma relação com a ocorrência (ou não) de efeitos contemporâneos de uma variável sobre outra (CAVALCANTI, 2010). 


\subsection{TESTE DE DEFASAGEM PELO CRITÉRIO DE AKAIKE (AIC)}

Realizado o teste de causalidade, é necessário determinar o número de defasagens do sistema por meio do chamado teste do coeficiente de máxima verossimilhança, que consiste na comparação de modelos com ordens de defasagens diferentes (Tabela 2). A aceitação da hipótese nula desse teste afirma que os modelos não possuem diferença, e o modelo escolhido é aquele que possui menor número de defasagens (ENDERS, 2004).

Com o objetivo de selecionar o melhor modelo, geralmente são utilizados os critérios de Akaike (AIC), Schwarz (SIC) e Hanna-Quinn (HQ). No presente estudo, realizou-se o teste de defasagem pelos critérios de Akaike (AIC).

Tabela 2 - Critério de seleção da ordem de defasagem do modelo - AIC/VAR

\begin{tabular}{|c|c|c|}
\hline Defasagem & AICC & Variáveis \\
\hline \multicolumn{3}{|c|}{ R.M. Recife } \\
\hline 1 & 1498,20 & $d \mathrm{ATV}-d \mathrm{SM}$ \\
\hline 1 & 1484,77 & $d \mathrm{INFO}-d \mathrm{SM}$ \\
\hline 1 & 1531,84 & $d \mathrm{DES}-d \mathrm{SM}$ \\
\hline \multicolumn{3}{|c|}{ R.M. Porto Alegre } \\
\hline 1 & 1399,33 & $d \mathrm{ATV}-d \mathrm{SM}$ \\
\hline 2 & 1428,72 & $d \mathrm{INFO}-d \mathrm{SM}$ \\
\hline 3 & 1384,46 & $d \mathrm{DES}-d \mathrm{SM}$ \\
\hline \multicolumn{3}{|c|}{ R.M Belo Horizonte } \\
\hline 1 & 1380,26 & $d \mathrm{ATV}-d \mathrm{SM}$ \\
\hline 1 & 1416,43 & $d \mathrm{INFO}-d \mathrm{SM}$ \\
\hline 4 & 1410,15 & $d \mathrm{DES}-d \mathrm{SM}$ \\
\hline \multicolumn{3}{|c|}{ R.M São Paulo } \\
\hline 1 & 1306,54 & $d \mathrm{ATV}-d \mathrm{SM}$ \\
\hline 1 & 1353,61 & $d \mathrm{INFO}-d \mathrm{SM}$ \\
\hline 4 & 1432,72 & $d \mathrm{DES}-d \mathrm{SM}$ \\
\hline
\end{tabular}

Fonte: Elaboração própria com base nos dados da pesquisa.

\subsection{ANÁLISE DA DECOMPOSIÇÃO DA VARIÂNCIA DO ERRO DE PREVISÃO}

A análise da decomposição da variância está entre os procedimentos que permitem o estudo das correlações entre um dado conjunto de variáveis expressas por um modelo VAR. Em outras palavras, esse procedimento explica quanto uma variável afeta a variância de outra variável. A Tabela 3 apresenta um resumo das magnitudes de influência captadas na presente análise.

A comparação dos resultados da análise de decomposição de variância da Tabela 1 indica que nas regiões metropolitanas do Recife, Porto Alegre, Belo Horizonte e São Paulo, o mercado de trabalho pouco é afetado pela política de salário mínimo. Sendo assim, variações na taxa de atividade, grau de informalidade e taxa de desemprego são praticamente explicadas pelo comportamento da própria variável. 
Tabela 3 - Resultados da decomposição da variância do erro (em \%)

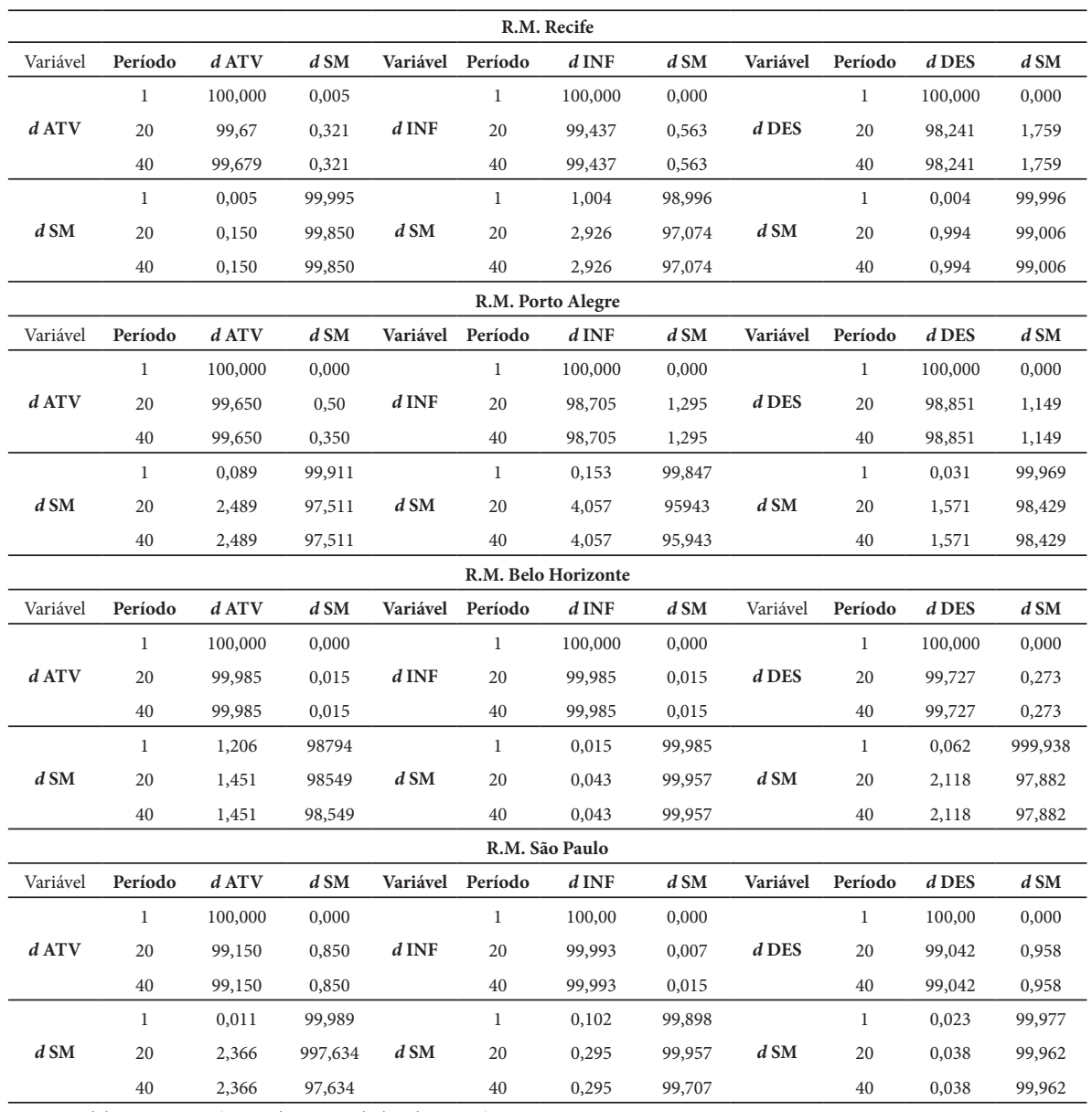

Fonte: Elaboração própria com base nos dados da pesquisa.

\subsection{ANÁLISE DAS FUNÇÕES DE RESPOSTA AOS IMPULSOS}

Os Gráficos 1, 2, 3 e 4 e a Tabela 4 apresentam a avaliação de respostas a impulso, considerando como um choque permanente no valor do salário mínimo real explica a trajetória das três demais variáveis.

\subsubsection{REGIÃO METROPOLITANA DO RECIFE}

Ao analisar os impactos que o salário mínimo real causa sobre as variáveis, observa-se, no Gráfico 1, uma trajetória semelhante para as três, em que predomina efeito negativo 
logo no primeiro período posterior ao choque, com o tempo de convergência se dando simultaneamente no quarto período, em que predomina uma queda maior na taxa de desemprego. A Tabela 4 mostra como as variáveis do mercado de trabalho nas regiões selecionadas se comportam com uma mudança relativa de $1 \%$ no salário mínimo.

$\mathrm{Na}$ região metropolitana de Recife, a maior resposta ao choque no salário nas taxas de atividade, grau de informalidade e taxa de desemprego é de $-0,26 \% ;-0,32 \%$ e $-0,69$, respectivamente. Pelo efeito acumulado, percebe-se o efeito de curto prazo, com maior queda da taxa de desemprego e maior entrada da mão de obra para o setor informal de trabalho, sobretudo no comércio e na prestação de serviços, principais setores da economia da região (DIEESE, 2015).

\section{Grafico 1 - Resposta da taxa de atividade, do grau de informalidade e da taxa de desemprego a choques no valor do salário mínimo real - R. M. Recife}
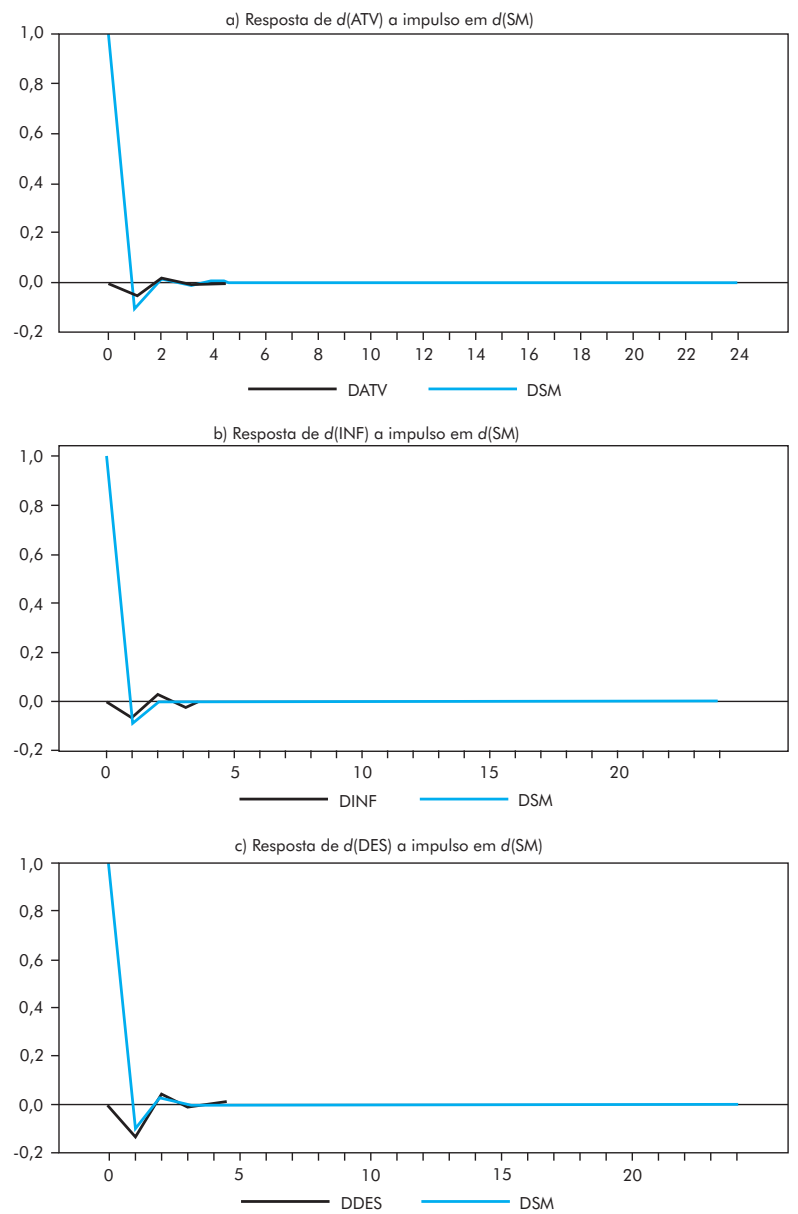

Fonte: Elaboração própria com base nos dados da pesquisa. 
Tabela 4 - Respostas acumuladas (em valores relativos) das variáveis selecionadas a um choque de $1 \%$ no salário mínimo - R.M. Recife

\begin{tabular}{lcccccc}
\hline Choque & $\begin{array}{c}\text { Resposta } \\
\text { Máxima }\end{array}$ & $\begin{array}{c}\text { Resposta } \\
\text { Acumulada } \\
\text { Máxima }\end{array}$ & $\begin{array}{c}\text { Resposta } \\
\text { Acumulada } \\
\text { para o 2 } \\
\text { período }\end{array}$ & $\begin{array}{c}\text { Resposta } \\
\text { Acumulada } \\
\text { para o 3 } \\
\text { período }\end{array}$ & $\begin{array}{c}\text { Resposta } \\
\text { Acumulada } \\
\text { para o 4 } \\
\text { período }\end{array}$ & $\begin{array}{c}\text { Resposta } \\
\text { Acumulada } \\
\text { para o 5 } \\
\text { período }\end{array}$ \\
\hline$d \mathrm{SM} \rightarrow d$ ATV & $-0,26$ & $-0,26$ & $-0,26$ & $-0,17$ & $-0,19$ & $-0,19$ \\
$d \mathrm{SM} \rightarrow d \mathrm{INF}$ & $-0,32$ & $-0,32$ & $-0,32$ & $-0,18$ & $-0,23$ & $-0,21$ \\
$d$ SM $\rightarrow d$ DES & $-0,69$ & $-0,69$ & $-0,69$ & $-0,49$ & $-0,54$ & $-0,21$ \\
\hline Fonte: Elaboração própria com base nos dados da pesquisa.
\end{tabular}

\subsubsection{REGIÃO METROPOLITANA DE PORTO ALEGRE}

No Gráfico 2, temos que na região metropolitana de Porto Alegre ocorre predominantemente um incremento da população economicamente ativa com deslocamento da mão de obra logo após o choque no valor do salário mínimo. Observa-se uma resposta positiva mais evidente para o setor informal de trabalho, onde esta foi positiva após o choque, com efeitos dissipando-se após o quarto período. Ainda verificando o gráfico, constata-se que um choque no valor do salário mínimo causa predominância de efeitos negativos na taxa de desemprego. Logo, trabalhadores demitidos no setor formal de trabalho se deslocam mais facilmente para a informalidade.

$\mathrm{Na}$ Tabela 5, com um resumo das respostas ao choque de $1 \%$, temos a confirmação da representação gráfica. Observa-se o crescimento da informalidade com apresentação da maior resposta a uma mudança relativa de $1 \%$ no salário mínimo, com $0,42 \%$ contra $0,19 \%$ e $-0,24 \%$ das taxas de atividade e desemprego, respectivamente. O impulso no setor informal mostrado nos resultados pode ocorrer, em grande medida, por grupo de indivíduos que já completaram seu ciclo produtivo e retornam ao mercado de trabalho motivados pela complementação da renda ou mesmo como um meio de socialização, onde a carteira de trabalho assinada não corresponde a aspecto que mais atrai para esse retorno (IPEA, 2015). 
Grafico 2 - Resposta da taxa de atividade, do grau de informalidade e da taxa de desemprego a choques no valor do salário mínimo real - R. M. Porto Alegre
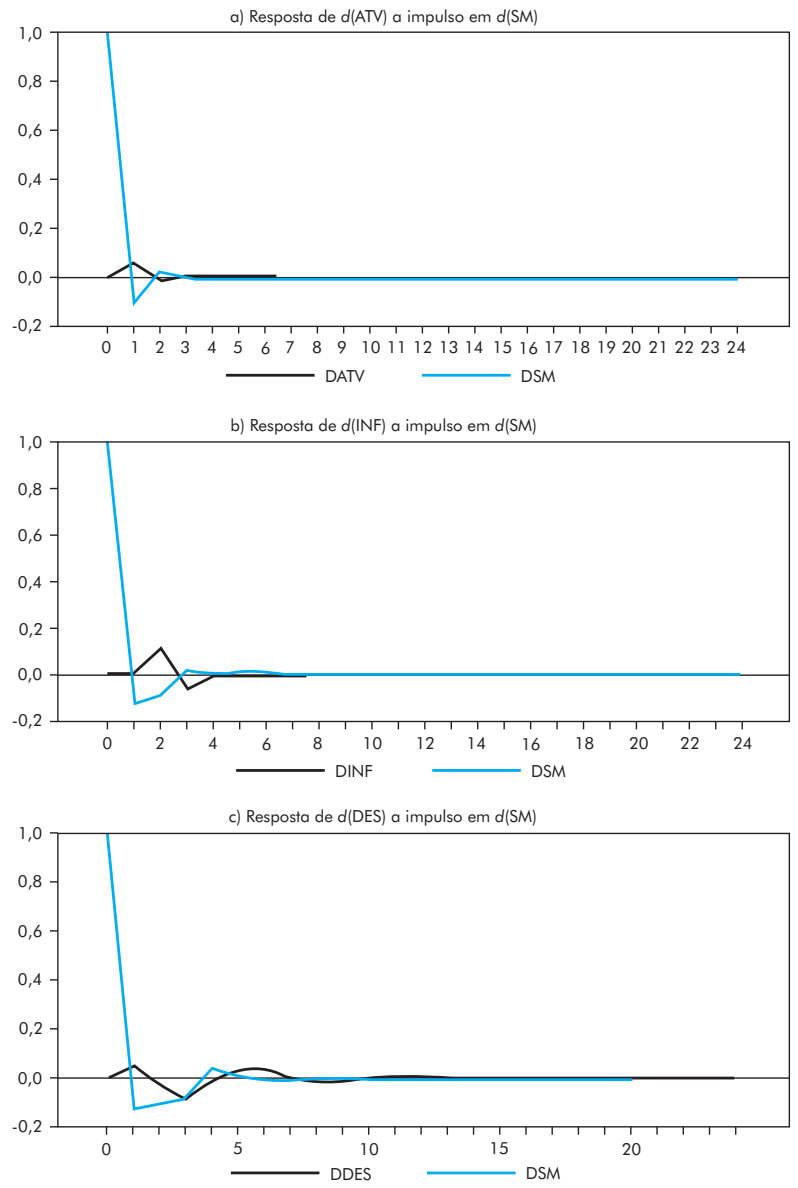

Fonte: Elaboração própria com base nos dados da pesquisa

Tabela 5 - Respostas acumuladas (em valores relativos) das variáveis selecionadas a um choque de $1 \%$ no salário mínimo - R.M. Porto Alegre

\begin{tabular}{ccccccc}
\hline Choque & $\begin{array}{c}\text { Resposta } \\
\text { Máxima }\end{array}$ & $\begin{array}{c}\text { Resposta } \\
\text { Acumulada } \\
\text { Máxima }\end{array}$ & $\begin{array}{c}\text { Resposta } \\
\text { Acumulada } \\
\text { para o 2 } \\
\text { período }\end{array}$ & $\begin{array}{c}\text { Resposta } \\
\text { Acumulada } \\
\text { para o 3 } \\
\text { período }\end{array}$ & $\begin{array}{c}\text { Resposta } \\
\text { Acumulada } \\
\text { para o 4o } \\
\text { período }\end{array}$ & $\begin{array}{c}\text { Resposta } \\
\text { Acumulada } \\
\text { para o 5 } \\
\text { período }\end{array}$ \\
\hline$d \mathrm{SM} \rightarrow d \mathrm{ATV}$ & 0,19 & 0,19 & 0,19 & 0,15 & 0,16 & 0,16 \\
$d \mathrm{SM} \rightarrow d \mathrm{INF}$ & 0,41 & 0,42 & 0,02 & 0,42 & 0,22 & 0,20 \\
$d \mathrm{SM} \rightarrow d \mathrm{DES}$ & $-0,27$ & $-0,24$ & 0,16 & 0,07 & $-0,20$ & $-0,24$ \\
\hline
\end{tabular}

Fonte: Elaboração própria com base nos dados da pesquisa. 


\subsubsection{REGIÃO METROPOLITANA DE BELO HORIZONTE}

Considerando a região metropolitana de Belo Horizonte (Gráfico 3), observa-se que o salário mínimo real causa mais impacto na taxa de desemprego. Verifica-se que esta apresentou resposta positiva após o choque no salário mínimo. Entretanto, após tal período, a resposta se dá de forma negativa, com o efeito se dissipando após o quarto período ao choque. O grau de informalidade e a taxa de atividade possuem predominância negativa, com efeitos semelhantes. Há, então, na região metropolitana de Belo Horizonte, aumento do fluxo de mão de obra, após uma elevação no valor do salário mínimo, para o estado de desemprego. No acumulado ao choque de $1 \%$ no salário (Tabela 6), a resposta positiva da taxa de desemprego corresponde a $0,24 \%$ com efeitos dissipando-se após o quinto período.

Grafico 3 - Resposta da taxa de atividade, do grau de informalidade e da taxa de desemprego a choques no valor do salário mínimo real - R. M. Belo Horizonte
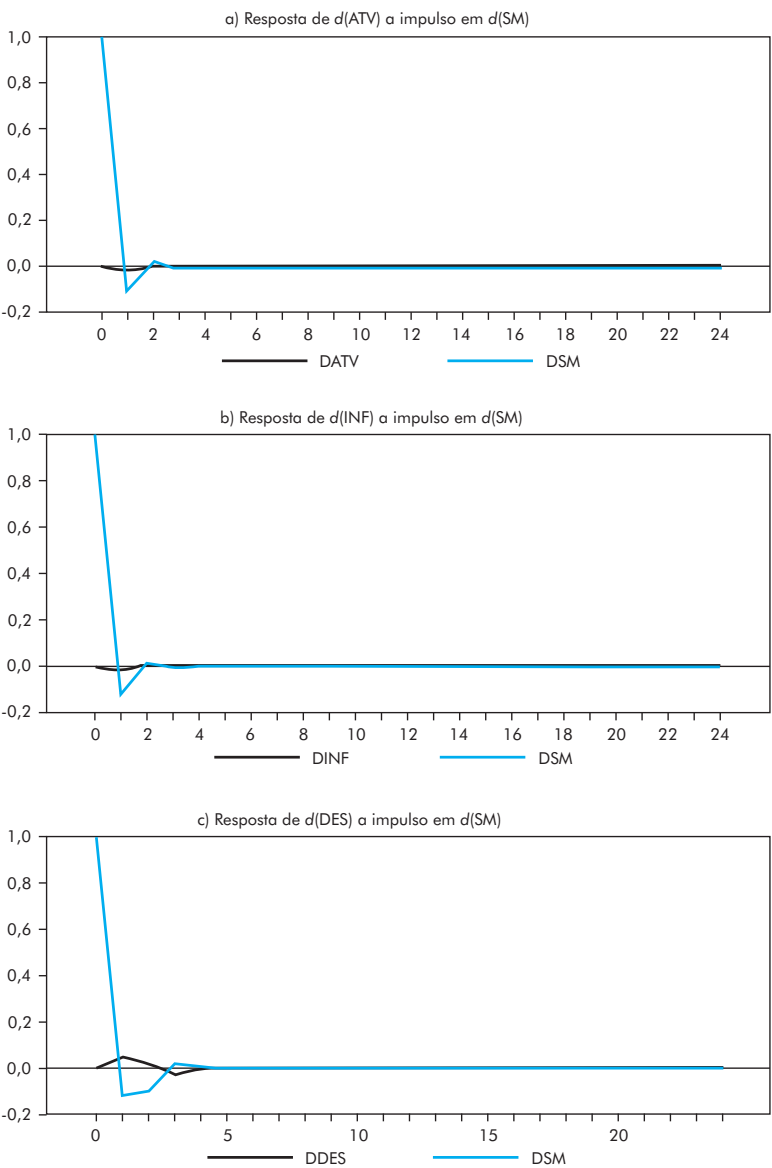

Fonte: Elaboração própria com base nos dados da pesquisa. 
Tabela 6 - Respostas acumuladas (em valores relativos) das variáveis selecionadas a um choque de $1 \%$ no salário mínimo - R. M. Belo Horizonte

\begin{tabular}{ccccccc}
\hline Choque & $\begin{array}{c}\text { Resposta } \\
\text { Máxima }\end{array}$ & $\begin{array}{c}\text { Resposta } \\
\text { Acumulada } \\
\text { Máxima }\end{array}$ & $\begin{array}{c}\text { Resposta } \\
\text { Acumulada } \\
\text { para o 2 } \\
\text { período }\end{array}$ & $\begin{array}{c}\text { Resposta } \\
\text { Acumulada } \\
\text { para o 3 } \\
\text { período }\end{array}$ & $\begin{array}{c}\text { Resposta } \\
\text { Acumulada } \\
\text { para o 4 } \\
\text { período }\end{array}$ & $\begin{array}{c}\text { Resposta } \\
\text { Acumulada } \\
\text { para o 5 }^{\mathbf{0}} \\
\text { período }^{\mathbf{0}}\end{array}$ \\
\hline$d \mathrm{SM} \rightarrow d \mathrm{ATV}$ & $-0,04$ & $-0,04$ & $-0,04$ & $-0,03$ & $-0,03$ & $-0,03$ \\
$d \mathrm{SM} \rightarrow d \mathrm{INF}$ & $-0,04$ & $-0,04$ & $-0,04$ & $-0,03$ & $-0,03$ & $-0,03$ \\
$d \mathrm{SM} \rightarrow d \mathrm{DES}$ & $-0,16$ & 0,24 & 0,16 & 0,24 & 0,15 & 0,15 \\
\hline
\end{tabular}

Fonte: Elaboração própria com base nos dados da pesquisa.

\subsubsection{REGIÃO METROPOLITANA DE SÃO PAULO}

Na região metropolitana de São Paulo (Gráfico 4), a resposta ao choque do salário mínimo ocorre de forma mais evidente nas variáveis taxa de atividade e taxa de desemprego. Na taxa de atividade, ocorre efeito predominantemete negativo. O setor informal também apresenta efeitos de tendência negativa, com implicações dissipando-se ainda nos primeiros períodos. Em contrapartida, observa-se que o deslocamento da mão de obra ocorre de forma significativamente positiva para o estado de desemprego, com resposta acumulada máxima, conforme a Tabela 7 , de $0,24 \%$ ao choque de $1 \%$ no salário contrastando com a resposta negativa de $-0,23 \%$ da taxa de atividade.

Temos nessa região que a elevação no salário mínimo não desloca mão de obra para o setor informal do mercado de trabalho. A resposta positiva da taxa de desemprego pode ser explicada pela redução da criação de novas vagas e a eliminação de postos de trabalho já existentes. Isso configuraria um impacto negativo sobre os salários, pois as empresas deverão promover maior rotatividade para se beneficiar do aumento da oferta de mão de obra. Ocorre com isso a substituição dos funcionários ativos, contratados quando o mercado estava aquecido, por novos, com salários menores (DIEESE, 2015).

Tabela 7 - Respostas acumuladas (em valores relativos) das variáveis selecionadas a um choque de $1 \%$ no salário mínimo - R. M. São Paulo

\begin{tabular}{|c|c|c|c|c|c|c|}
\hline Choque & $\begin{array}{l}\text { Resposta } \\
\text { Máxima }\end{array}$ & $\begin{array}{c}\text { Resposta } \\
\text { Acumulada } \\
\text { Máxima }\end{array}$ & $\begin{array}{c}\text { Resposta } \\
\text { Acumulada } \\
\text { para o 2o } \\
\text { período }\end{array}$ & $\begin{array}{c}\text { Resposta } \\
\text { Acumulada } \\
\text { para o } 3^{\circ} \\
\text { período }\end{array}$ & $\begin{array}{c}\text { Resposta } \\
\text { Acumulada } \\
\text { para o } 4^{\circ} \\
\text { período }\end{array}$ & $\begin{array}{c}\text { Resposta } \\
\text { Acumulada } \\
\text { para o } 5^{\circ} \\
\text { período }\end{array}$ \\
\hline$d \mathrm{SM} \rightarrow d \mathrm{ATV}$ & $-0,23$ & $-0,23$ & $-0,23$ & $-0,18$ & $-0,18$ & $-0,18$ \\
\hline$d \mathrm{SM} \rightarrow d \mathrm{DES}$ & 0,38 & 0,38 & 0,38 & 0,36 & 0,37 & 0,37 \\
\hline
\end{tabular}

Fonte: Elaboração própria com base nos dados da pesquisa. 
Grafico 4 - Resposta da taxa de atividade, do grau de informalidade e da taxa de desemprego a choques no valor do salário mínimo real - R. M. São Paulo
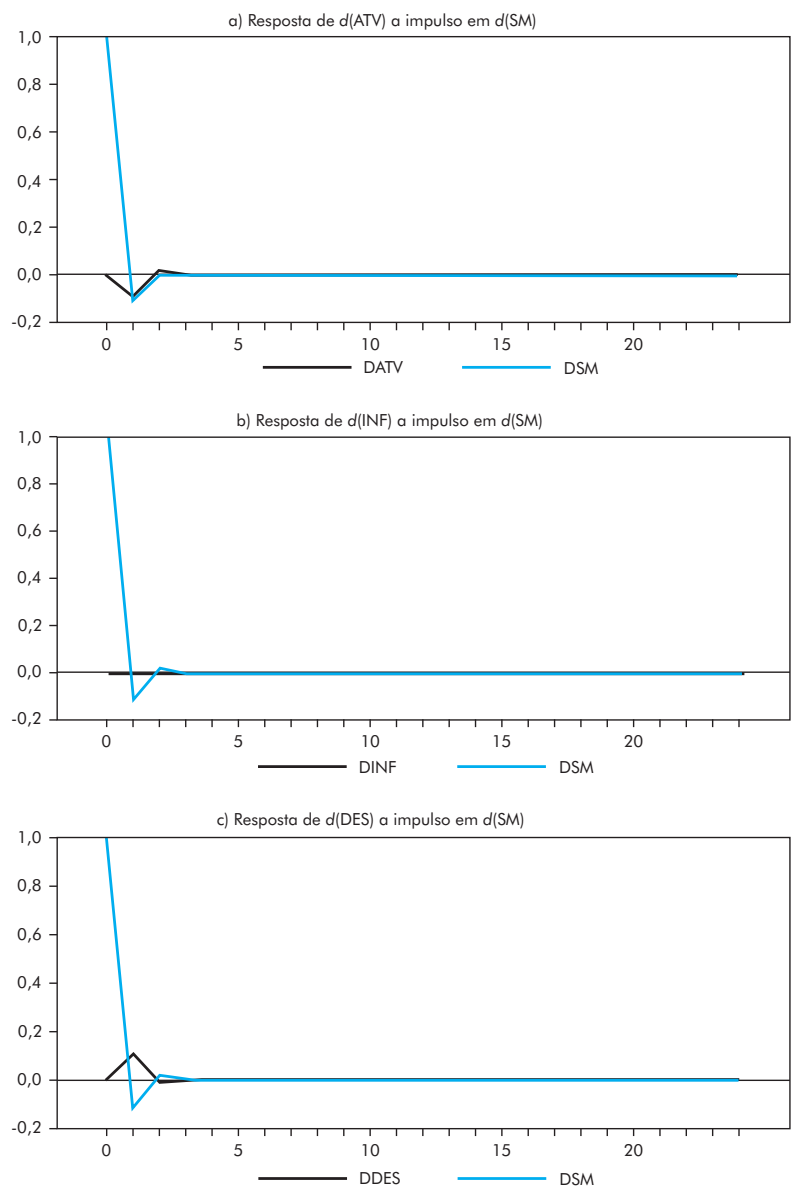

Fonte: Elaboração própria com base nos dados da pesquisa.

\section{CONSIDERAÇÕES FINAIS}

Tomando o salário mínimo como o preço da mão de obra pouco qualificada, mudanças no seu valor alteram os salários relativos dos trabalhadores e ocasionam um aumento de custos para as empresas. Nesse sentido, suas elevações tendem a provocar ajustes, os quais podem levar a modificações significativas no nível de emprego, na qualificação média da mão de obra, na taxa de desemprego e no nível de lucros. Além disso, uma vez que sua legislação nem sempre é cumprida, aumentos no salário mínimo podem provocar efeitos sobre o grau de informalidade. Na realidade, é essencialmente a partir da 
combinação desses efeitos que o salário mínimo provoca alterações sobre a estrutura de renda e emprego da economia (SOUZA e TARGINO, 2005).

Por meio do modelo VAR, procurou-se a obtenção do comportamento de variáveis do mercado de trabalho a choques de salário mínimo. Nas regiões consideradas, a elevação no valor do salário mínimo não causa efeito de longo prazo em todas as variáveis. Em todas as regiões metropolitanas observa-se a entrada de mão de obra em idade ativa no mercado de trabalho. Sabendo que a taxa de atividade representa o quanto da população em idade ativa está inserida no mercado de trabalho, pode-se interpretar seu comportamento como uma relação positiva entre salário e oferta de trabalho, a qual se dá em conformidade com a curva de oferta de trabalho positivamente inclinada.

Os resultados, quando o salário mínimo se relaciona com o grau de informalidade, apresentam algumas distinções. Há uma queda maior comparando-se com a taxa de atividade nas regiões metropolitanas de Recife, Belo Horizonte e São Paulo. Na região metropolitana de Porto Alegre, observa-se a tendência de deslocamento da mão de obra no setor formal para a informalidade. Os resultados retratam a ambiguidade presente nos modelos teóricos que tratam da resposta do emprego informal à elevação no salário mínimo, podendo ocorrer migração de trabalhadores do setor formal para o informal.

Entretanto, outros trabalhadores que perderam o emprego no setor formal podem decidir não trabalhar no setor informal, podendo se tornar desempregados à espera de uma ocupação no setor formal ou, então, saírem da força de trabalho. Para o modelo competitivo padrão, temos que a imposição do salário mínimo faz surgir um excedente de oferta em relação à demanda, implicando no incremento das taxas de desemprego (IPEA, 2015). Tal resultado pode ser bem evidenciado nas regiões metropolitanas de Belo Horizonte e São Paulo, com o crescimento dessa taxa.

Além de condicionantes para a mudança no nível de emprego nas regiões como a pouca qualificação do trabalho, as crises econômicas, a substituição da mão de obra humana por máquinas e o custo alto parta a contratação de funcionários com carteira assinada, o aumento de taxas de desemprego também é incrementado a partir do momento em que este não é caracterizado apenas pela ausência do indivíduo no mercado de trabalho, mas também pela pressão que faz nesse mercado.

Resultados negativos acerca da repercussão do aumento no salário mínimo sobre a taxa de desemprego se dão de forma mais relevante na região metropolitana de Recife, que apresenta uma migração menor da mão de obra para o estado de desemprego após choques no salário mínimo, muito embora a região de Porto Alegre também venha a apresentar tendência negativa, com alternâncias de períodos de entrada de mão de obra para o estado de desemprego. 


\section{REFERÊNCIAS}

BARROS, R. P. de; CARVALHO, M. Salário mínimo e distribuição de renda. Seminários DIMAC, $\mathrm{n}^{\circ}$ 196, 2005.

BOERI, T.; GARIBALDI P.; RIBEIRO M. Behind the lighthouse effect. IZA Discussion Paper, Institut zur Zukunft der Arbeit (IZA - Centro de Estudos sobre o Futuro do Trabalho), n. $4890,2010$.

CACCIAMALI, M. C. "Pode o salário mínimo balizar a estrutura salarial das firmas? Argumentos a favor da política ativa de salário mínimo". In: BALTAR, P.; DEDECCA, C.; KREIN, J. Salário mínimo e desenvolvimento. Campinas, São Paulo: Instituto de Economia/Unicamp, 2005, p. 147-162.

CAVALCANTI M. A. F. H. Identificação de modelos VAR e causalidade de Granger: uma nota de advertência. Economia Aplicada, v. 14, n. 2, p. 251-260, 2010.

DICKEY, D. A.; FULLER, W. Likelihood ratio statistics for autoregressive time series with a unit root. Econometrica, v. 49, n. 4, p. 1057-1079, jul. 1981.

DIEESE - DEPARTAMENTO INTERSINDICAL DE ESTATÍSTICAS E ESTUDOS SOCIOECONÔMICOS. Boletim de Conjuntura do Departamento Intersindical de Estatística e Estudos Socioeconômico, n. 4, ago. 2015. Disponível em: <http://www.dieese.org.br/boletimdeconjuntura/2015/boletimConjuntura004.pdf>. Acesso em: 1 out. 2015.

ENDERS, W. Rats Handbook for Econometric Time Series. Nova York: John Wiley \& Sons, 1995.

ENDERS, W. Applied Econometric Time Series. 2 ed. New York: Wiley, 2004.

ENGLE, R. F.; GRANGER, C. W. J.. Cointegration and Error-correction: representation, estimation, and testing. Econometrica, n. 55, p. 251-276, mar. 1987. Disponível em <http://www. uta.edu/faculty/crowder/papers/Engle_Granger_1987.pdf> Acesso em: 3 out. 2015.

FOGUEL, M.; ULYSSEA, G.; COURSEIL, C. H. "Salário mínimo e mercado de trabalho no Brasil”. In: MONASTERIO, L. M.; NERI, M. C.; SOARES, S. S. D. (Orgs.). Brasil em desenvolvimento 2014: estado, planejamento e políticas públicas - vol. 1. Brasília: Ipea, 2014.

FREITAS, P. S. “Salário mínimo e mercado de trabalho no Brasil”. In: MENEGUIN, F. B. Agenda Legislativa para o Desenvolvimento Nacional. Brasília: Senado Federal. Subsecretaria de Edições Técnicas, 2011.

GANDRA, R. M. O debate sobre a desigualdade de renda no Brasil: da controvérsia dos anos 70 ao pensamento hegemônico nos anos 90. Texto para Discussão, IE-UFRJ, n. 1, 2004.

GRAMLICH, E. Impact of minimum wages on other wages, employment and family incomes. Brookings Papers on Economy Activity, v. 7, p. 409-451, 1976.

GRANGER, C. Investigating causal relations by econometric models and cross-spectral methods. Econometrica, n. 37, p. 424-438, 1969.

GIAMBIAGI, F; FRANCO, S. Esgotamento do papel do salário mínimo como mecanismo de combate à pobreza extrema. Texto para Discussão, IPEA, Rio de Janeiro, n. 1290, 2007.

HICKS, J. R. Mr. Keynes theory and classics. Econometrica, abr. 1937. 
IBGE - INSTITUTO BRASILEIRO DE GEOGRAFIA E ESTATÍSTICA. Pesquisa Mensal do Emprego. Estimativas para o mês de março de 2015 nas regiões metropolitanas de Recife, Porto Alegre, Belo horizonte e São Paulo. Rio de Janeiro: IBGE, 2015.

IPEA - INSTITUTO DE PESQUISA ECONÔMICA APLICADA. Boletim Mercado de Trabalho - Conjuntura e Análise, n. 59, 2015.

LEMOS, S. The effect of the minimum wage on prices. IZA Discussion Paper, Institut zur Zukunft der Arbeit (IZA - Centro de Estudos sobre o Futuro do Trabalho), n. 1.072, 2004.

MACEDO, R. B. M.; GARCIA, M. E. Observações sobre a política brasileira de salário mínimo. Trabalho para Discussão, IPE/FEA-USP, 1978.

MINCER, J. Unemployment effects of minimun wage. NBER Working Papers, n. 0039, 1976.

MOURA, R. L; NERI, M. C. Efetividade do salário mínimo estadual: Uma análise via regressões quantílicas para dados longitudinais. Revista de Economia Aplicada, v. 12, n. 2, p. 239-273, abr./jun. 2008.

ROMANNATTO, E.; PORCILE, G.; CURADO, M. Produtividade, salários e taxa de câmbio: uma análise da experiência brasileira nos anos 1990. Revista Economia Contemporânea, Rio de Janeiro, v. 12, n. 3, p. 545-570, set./dez. 2008.

SOUZA, G. L.; TARGINO, I. Política de salário mínimo e seus efeitos sobre o mercado de trabalho metropolitano. Revista Economia e Desenvolvimento, Recife-PE, v. 4, n. 2, p. 299-334, 2005.

SIMS, C. Macroeconomics and reality. Econometrica, Illinois, v. 48, n. 1, p. 1-48, jan. 1980.

SOARES, S. S. D. O impacto distributivo do salário mínimo: a distribuição individual dos rendimentos do trabalho. Economia Aplicada, v. 8, n. 1, p. 47-76, 2004.

STADUTO, J. A. R. Determinação dos salários na agropecuária brasileira - período de 1971 a 1966. Tese (Doutorado em Economia Aplicada) - Escola Superior de Agricultura Luiz de Queiroz, Universidade de São Paulo, São Paulo, 2002.

WELCH, F. Minimum Wage Legislation in the United States. Economy Inquiry, cap. 12, p. 235 318,1974 


\section{ANEXOS}

Tabela I - Teste de cointegração de Engle-Granger

\begin{tabular}{|c|c|c|c|c|}
\hline \multicolumn{5}{|c|}{ R. M. do Recife } \\
\hline Variável & Coeficiente & Desvio Padrão & $\mathrm{T}$ - Estatística & Significância \\
\hline \multicolumn{5}{|c|}{$d$ ATV - Variável dependente } \\
\hline$d \mathrm{ATV}$ & $-0,2684$ & 0,0805 & $-3,3347$ & 0,0011 \\
\hline$d \mathrm{SM}$ & $-0,0010$ & 0,0032 & $-0,3141$ & 0,7539 \\
\hline \multicolumn{5}{|c|}{$d$ INFO - Variável dependente } \\
\hline$d$ INF & $-0,3584$ & 20,0790 & $-4,5188$ & 0,0000 \\
\hline$d \mathrm{SM}$ & $-0,0021$ & 0,0031 & $-0,6791$ & 0,4982 \\
\hline \multicolumn{5}{|c|}{$d$ DES - Variável dependente } \\
\hline$d$ DESD & $-0,1966$ & 0,0817 & $-2,4081$ & 0,0173 \\
\hline$d \mathrm{SM}$ & $-0,0060$ & 0,0037 & $-1,6234$ & 0,1067 \\
\hline \multicolumn{5}{|c|}{ R. M. de Porto Alegre } \\
\hline Variável & Coeficiente & Desvio Padrão & $\mathrm{T}$ - Estatística & Significância \\
\hline \multicolumn{5}{|c|}{$d$ ATV - Variável dependente } \\
\hline$d$ ATV & $-0,1031$ & 0,0827 & $-1,2468$ & 0,2145 \\
\hline$d \mathrm{SM}$ & 0,0026 & 0,0023 & 1,1250 & 0,2625 \\
\hline \multicolumn{5}{|c|}{$d$ INFO - Variável dependente } \\
\hline$d$ INF & $-0,1465$ & 0,0827 & $-1,2468$ & 0,2145 \\
\hline$d \mathrm{SM}$ & 0,0026 & 0,0023 & 1,1250 & 0,2625 \\
\hline \multicolumn{5}{|c|}{$d$ DES - Variável dependente } \\
\hline$d$ DESD & $-0,3167$ & 0,0814 & $-3,8897$ & 0,0002 \\
\hline$d \mathrm{SM}$ & $-0,0014$ & 0,0022 & $-0,6066$ & 0,5452 \\
\hline \multicolumn{5}{|c|}{ R. M. de Belo Horizonte } \\
\hline Variável & Coeficiente & Desvio Padrão & $\mathrm{T}$ - Estatística & Significância \\
\hline \multicolumn{5}{|c|}{$d$ ATV - Variável dependente } \\
\hline$d$ ATV & $-0,2485$ & 0,0817 & $-3,0419$ & 0,0028 \\
\hline$d \mathrm{SM}$ & 0,0007 & 0,0023 & 0,3049 & 0,7609 \\
\hline \multicolumn{5}{|c|}{$d$ INFO - Variável dependente } \\
\hline$d$ INF & $-0,1705$ & 0,0827 & $-2,0630$ & 0,0409 \\
\hline$d \mathrm{SM}$ & 0,0000 & 0,0024 & 0,0154 & 0,9878 \\
\hline \multicolumn{5}{|c|}{$d$ DES - Variável dependente } \\
\hline$d$ DESD & $-0,0945$ & 0,0841 & $-1,1244$ & 0,2629 \\
\hline$d \mathrm{SM}$ & 0,0063 & 0,0025 & 2,4909 & 0,0140 \\
\hline \multicolumn{5}{|c|}{ R. M. de São Paulo } \\
\hline Variável & Coeficiente & Desvio Padrão & T - Estatística & Significância \\
\hline \multicolumn{5}{|c|}{$d$ ATV - Variável dependente } \\
\hline$d$ ATV & $-0,1138$ & 0,0833 & $-1,3664$ & 0,1740 \\
\hline$d \mathrm{SM}$ & $-0,0013$ & 0,0017 & 0,7804 & 0,4365 \\
\hline \multicolumn{5}{|c|}{$d$ INFO - Variável dependente } \\
\hline$d$ INF & $-0,2545$ & 0,0822 & $-3,0966$ & 0,0024 \\
\hline$d \mathrm{SM}$ & $-0,0017$ & 0,0020 & $-0,8496$ & 0,3970 \\
\hline \multicolumn{5}{|c|}{$d$ DES - Variável dependente } \\
\hline$d$ DESD & $-0,1475$ & 0,0853 & $-1,7262$ & 0,0866 \\
\hline$d \mathrm{SM}$ & 0,0018 & 0,0027 & 0,6725 & 0,5024 \\
\hline
\end{tabular}

Fonte: Elaboração própria com base nos dados da pesquisa. 